\title{
Parental knowledge about familial Mediterranean fever: a cross-sectional study
}

\author{
Deniz Gezgin Yıldırım ${ }^{1 \oplus}$, Sevcan A. Bakkaloğlu' ${ }^{1 \oplus}$, A. Şebnem Soysal Acar² \\ Necla Buyan ${ }^{1 \odot}$ \\ ${ }^{1}$ Division of Pediatric Rheumatology, ${ }^{2}$ Department of Pediatrics, Gazi University Faculty of Medicine, Ankara, Turkey.
}

\begin{abstract}
Background. The life-long course, long-term complications, necessity for regular treatment, and potential side effects of the medications must be well understood by parents of pediatric familial Mediterranean fever (FMF) patients. The aim of this study was to assess parental knowledge and to investigate how parents obtained scientific information about FMF.
\end{abstract}

Methods. One hundred and seventy-one pediatric FMF patients and their parents were enrolled in this crosssectional study. Three-part questionnaires, including forms on socio-demographics, knowledge and perceptions of FMF, and how to get information about FMF, were administered to parents.

Results. In the analysis of the knowledge questions, $90.1 \%$ of parents were aware of colchicine as an effective drug for FMF, but only $39.2 \%$ of them were aware that there is no vital risk during FMF attacks. Caregivers preferred to obtain information from physicians (98.8\%), websites (47.9\%), seminars (3.5\%), and books (1.7\%). The knowledge scores of parents were significantly higher among those whose children were using antiinterleukin- 1 therapy in addition to colchicine relative to those on colchicine alone $(p=0.04)$. There was a positive correlation between knowledge level and parental educational status $(\mathrm{p}=0.0001)$.

Conclusions. Knowledge scores among parents of pediatric FMF patients are unsatisfactory. The parents whose children have a severe disease course and a need for anti-interleukin-1 therapy are more knowledgeable. For parents, continuing education programs including books, seminars and web-sites giving information about the course, prognosis, complications and treatments of FMF should be employed immediately after the diagnosis and thereafter.

Key words: childhood, familial Mediterranean fever, pediatric rheumatology, parental knowledge.

Familial Mediterranean fever (FMF) is the most common monogenic, chronic autoinflammatory disease characterized by recurrent attacks of fever, pleuritis, pericarditis, peritonitis and arthritis. The attacks are self-limiting and typically resolve within 24-72 hours. $^{1}$ Mediterranean fever (MEFV) gene mutation causes hyperactivity of inflammasomes which leads to an increase of interleukin-1 $\beta$ (IL-1 $\beta$ ) and resultant severe inflammation. ${ }^{2}$ The prevalence of FMF is changing among communities, and

$凶$ Deniz Gezgin Yıldırım

gezgindeniz@gmail.com

Received 16th September 2020, revised 5th December 2020, 23rd December 2020, accepted 6th April 2021. the disease is reported in approximately $1 / 1000$ people in Turkey. ${ }^{3}$ Colchicine is a cheap, well tolerated, and life-long treatment that prevents the development of amyloidosis and must be used daily by oral delivery. ${ }^{4}$ The inflammatory attacks generally occur before 20 years of age in $90 \%$ of FMF patients, so patients are generally diagnosed with FMF in childhood. ${ }^{5}$ As a result, parents take responsibility for their children to take regular colchicine every day and go to routine outpatient follow-up visits. ${ }^{5}$ Therefore, having a life-long disease with longterm complications, the necessity for life-long regular treatment, and the side effects of the therapy must be well understood by parents of pediatric FMF patients. Treatment compliance 
of pediatric FMF patients might be affected by social and demographic factors, such as education, age, and social network of parents. Parental knowledge about chronic diseases, such as rheumatic diseases, epilepsy, thalassemia, neurofibromatosis, and hypospadias, has been investigated previously. ${ }^{6-11}$ However, to date, no data has been reported about the parental knowledge of pediatric patients with FMF.

We, herein, aimed to evaluate parental knowledge and perceptions of FMF and to investigate how parents obtained scientific information about it.

\section{Material and Methods}

FMF patients aged 4-16 years who had been suffering from FMF for six months or more and their primary caregivers were asked to participate in this cross-sectional study by a pediatric rheumatologist and a social worker. All patients were being followed by the Pediatric Rheumatology Department at the Gazi University Faculty of Medicine between June 2018 and November 2018. Patients who had an additional chronic disease were excluded from the study. All patients were evaluated clinically according to the Tel Hashomer Criteria. ${ }^{12}$ Demographic data of patients and parents were recorded. All patients were receiving colchicine treatment. Resistance to colchicine therapy was quantified as experiencing one or more attacks per month despite receiving the maximally tolerated dose of colchicine for $\geq 6$ months. Partial response to colchicine therapy was accepted as a decrease in attack frequency. Complete response to colchicine was accepted as resolved inflammatory attacks and serum acute phase reactant levels. ${ }^{13}$ FMF patients using maximum tolerated colchicine dose regularly in everyday without forgetting were accepted as good compliance, while FMF patients with missing colchicine doses were accepted as noncompliance to the therapy. Biologics targeting IL-1 were started with some FMF patients due to partial response, resistant, non-compliance or intolerance to colchicine, or secondary amyloidosis development.
Pras activity scores were used for evaluating disease severity in FMF patients. ${ }^{14}$ The FMF severity score comprised of age at FMF onset, frequency of attacks, presence of arthritis, erysipelas-like erythema, amyloidosis, and the required dose of colchicine prophylaxis necessary to control FMF symptoms. Escalating scores indicate mild (score, 1-5), moderate (score, $6-9$ ), and severe (score, $>10$ ) FMF activity. ${ }^{15}$

Three self-administered surveys which were developed by authors, were given to caregivers:

a. Caregivers' socio-demographics form: Caregivers (mother or father) were asked to provide personal demographic information, including age, gender, education status, and having FMF or not.

b. FMF parental knowledge and perceptions form: The knowledge section of the questionnaire contained 14 items. To evaluate the parents' knowledge level of FMF, we posed a set of 14 questions to all participants. This non-standardized questionnaire was generated by authors. These questions included whether FMF is a contagious or a genetic disease or a disease that should be followed-up regularly in a pediatric rheumatology department; whether or not children with FMF have a vital risk during attacks or have lower IQs than their peers; whether or not colchicine is an effective treatment agent; whether or not drugs for FMF are addictive or have side effects such as infertility; whether or not FMF resolves spontaneously over time or worsens with age; whether or not FMF patients can work actively and join sport activities; and whether or not FMF symptoms get worse or irreversible damage develops in internal organs, such as kidneys, when medication is not used regularly (Table II). Each item is rated on a 3-point scale (Yes, No, I don't know). Each correct answer was given 1 point. The total score of knowledge was graded between 0 (the lowest grade) and 14 (the highest grade) points. 
had a positive family history for FMF. The median Pras activity score was 6 (3-15).

All knowledge-related questions are presented in Table II. Of 171 parents, 154 (90.1\%) knew that colchicine is an effective drug in FMF treatment. In contrast, only $67(39.2 \%)$ knew that there is not a vital risk during attacks of FMF disease.

In evaluating parents' sources for gathering knowledge about FMF, we determined they preferred obtaining information from physicians (98.8\%), web-sites $(47.9 \%)$, seminars $(3.5 \%)$, and books (1.7\%) (Table III).

Parental knowledge scores and patients' Pras activity scores were compared by demographic findings and treatment responses (Table IV). There were no significant differences in knowledge scores of parents by either patients' or parents' age, gender, education status, or compliance with colchicine use ( $\mathrm{p}>$ 0.05). However, the knowledge scores were significantly higher in parents whose children needed to use anti-IL-1 therapy in addition to colchicine $(p=0.04)$. There were no correlations between parental knowledge scores and Pras activity scores in mothers' ages, fathers' ages, patients' ages and colchicine compliance $(\mathrm{p}>$ 0.05) (Table V). There was, however, a positive correlation in knowledge level and parental educational status $(\mathrm{p}=0.0001)$.

Table II. Percentage of correct answers to questions comprising the knowledge score of parents of children with familial Mediterranean fever.

\begin{tabular}{lc}
\hline Items & $\begin{array}{c}\text { \% of correct } \\
\text { answers }\end{array}$ \\
\hline FMF is a contagious disease & 85.4 \\
FMF is a hereditary genetic disease & 71.3 \\
There is a vital risk during attacks of FMF disease & 39.2 \\
Colchicine is the effective drug in FMF treatment & 90.1 \\
Drugs of FMF are addictive & 49.7 \\
FMF is a disease that affects intelligence & 63.2 \\
FMF is a disease that passes by itself over time & 62.0 \\
FMF gets worse with age & 46.8 \\
FMF patients cannot work actively & 68.4 \\
FMF patients cannot do any sports & 74.3 \\
FMF disease gets worse when colchicine is not used regularly & 71.9 \\
Medications of FMF have side effects such as infertility & 61.4 \\
Damage to internal organs such as kidneys can develop when colchicine is not taken regularly & 83.6 \\
FMF is a chronic disease that must be followed-up regularly by a pediatric rheumatologist and/or & 80.1 \\
a pediatrician.
\end{tabular}

FMF: familial Mediterranean fever.

Table III. Parents' sources of information for familial Mediterranean fever.

\begin{tabular}{lc}
\hline Item & $\%$ \\
\hline I have information about FMF from physicians & 98.8 \\
I have read a book about FMF & 1.7 \\
I have participated a seminar about FMF & 3.5 \\
I have followed a web-site about FMF & 47.9 \\
\hline
\end{tabular}

FMF: familial Mediterranean fever. 
Table IV. Demographic findings and treatment responses by parental knowledge and by patients' Pras activity scores. ${ }^{12}$

\begin{tabular}{|c|c|c|c|c|}
\hline Factor & Knowledge & $\mathrm{p}$ value & Pras activity score ${ }^{*}$ & $\mathrm{p}$ value \\
\hline \multicolumn{5}{|l|}{ Parents } \\
\hline Mother & $8.9 \pm 2.9$ & 0.36 & $7.14 \pm 2.4$ & 0.20 \\
\hline Father & $9.5 \pm 2.1$ & & $6.69 \pm 2.3$ & \\
\hline \multicolumn{5}{|l|}{ Mother's age } \\
\hline 30-50 years & $8.9 \pm 2.7$ & 0.18 & $7.05 \pm 2.4$ & \\
\hline $51-70$ years & $10.1 \pm 3.1$ & & $7.19 \pm 2.3$ & \\
\hline \multicolumn{5}{|l|}{ Father's age } \\
\hline 30-50 years & $8.8 \pm 2.8$ & 0.17 & $7.08 \pm 2.5$ & 0.89 \\
\hline $51-70$ years & $9.6 \pm 2.6$ & & $7.02 \pm 2.3$ & \\
\hline \multicolumn{5}{|l|}{ Mother's education status } \\
\hline Primary school & $8.9 \pm 2.7$ & 0.43 & $6.54 \pm 1.9$ & 0.04 \\
\hline Secondary/high school/university & $9.3 \pm 2.8$ & & $7.33 \pm 2.6$ & \\
\hline \multicolumn{5}{|l|}{ Father's education status } \\
\hline Primary school & $8.9 \pm 2.9$ & 0.53 & $6.62 \pm 2.1$ & 0.012 \\
\hline Secondary/high school/university & $9.1 \pm 2.7$ & & $7.53 \pm 2.6$ & \\
\hline \multicolumn{5}{|l|}{ Patients with FMF } \\
\hline \multicolumn{5}{|l|}{ Age } \\
\hline 4-7 years & $8.7 \pm 3.1$ & 0.49 & $6.74 \pm 1.7$ & 0.33 \\
\hline 8-16 years & $9.1 \pm 2.6$ & & $7.17 \pm 2.6$ & \\
\hline \multicolumn{5}{|l|}{ Age at diagnosis } \\
\hline$<10$ years & $8.8 \pm 2.9$ & 0.22 & $7.27 \pm 2.4$ & 0.03 \\
\hline$>10$ years & $9.5 \pm 2.2$ & & $6.45 \pm 2.3$ & \\
\hline \multicolumn{5}{|l|}{ Compliance of colchicine use } \\
\hline Regular & $8.9 \pm 2.8$ & 0.53 & $7.18 \pm 2.5$ & 0.43 \\
\hline Not regular & $9.3 \pm 2.4$ & & $6.63 \pm 2.0$ & \\
\hline \multicolumn{5}{|l|}{ Response to colchicine } \\
\hline Complete response & $8.8 \pm 2.4$ & 0.04 & $6.73 \pm 2.3$ & 0.001 \\
\hline Incomplete response & $10.1 \pm 2.8$ & & $9.94 \pm 1.5$ & \\
\hline \multicolumn{5}{|l|}{ Anti-IL-1 therapy } \\
\hline Not users & $8.9 \pm 2.4$ & 0.04 & $6.73 \pm 2.2$ & 0.001 \\
\hline Users & $10.1 \pm 2.7$ & & $10.38 \pm 1.9$ & \\
\hline
\end{tabular}

FMF: familial Mediterranean fever, anti-IL-1: anti-interleukin-1.

*The Pras activity score evaluates the severity of the disease with scores of 2-5 for those having mild activity, 6-10 for moderate activity, and $>10$ for severe activity. ${ }^{15}$

\section{Discussion}

This study has evaluated parental knowledge, perceptions, and the ways parents access scientific information about FMF. This study showed that parents of FMF children did not have adequate knowledge about FMF, and they mostly tried to get information from their physicians and web-sites. Furthermore, we found that the parents whose children had a severe disease course and needed to use antiIL-1 therapy in addition to colchicine were more knowledgeable than the others about FMF disease. Also, the level of educational status of parents was positively correlated with knowledge about FMF disease. Surprisingly, the knowledge level of parents was insufficient 
Table V. Correlation between parental knowledge and patients' Pras activity scores

\begin{tabular}{|c|c|c|c|c|}
\hline \multirow{2}{*}{ Factor } & \multicolumn{2}{|c|}{ Knowledge } & \multicolumn{2}{|c|}{ Pras activity score* } \\
\hline & r value & $p$ value & r value & $\mathrm{p}$ value \\
\hline Child age & 0.12 & 0.09 & 0.04 & 0.55 \\
\hline Mother age & 0.13 & 0.09 & 0.01 & 0.87 \\
\hline Father age & 0.11 & 0.10 & 0.06 & 0.43 \\
\hline Age of onset symptoms & 0.13 & 0.09 & -0.38 & 0.0001 \\
\hline Attack frequency in a year & 0.28 & 0.0001 & 0.44 & 0.0001 \\
\hline Mother education & 0.35 & 0.0001 & -0.16 & 0.03 \\
\hline Father education & 0.33 & 0.0001 & -0.20 & 0.008 \\
\hline Response of colchicine treatment & -0.14 & 0.04 & 0.40 & 0.0001 \\
\hline Use of anti-IL-1 treatment & -0.07 & 0.36 & 0.29 & 0.0001 \\
\hline Compliance to colchicine treatment & 0.04 & 0.58 & -0.09 & 0.24 \\
\hline
\end{tabular}

Anti-IL-1: anti-interleukin-1.

*The Pras activity score evaluates the severity of the disease with scores of 2-5 for those having mild activity, 6-10 for moderate activity, and $>10$ for severe activity. ${ }^{15}$

even though Turkey is one of countries where FMF is most commonly seen.

Al-Eid et al. ${ }^{7}$ reported that the majority of parents have insufficient knowledge regarding rheumatic diseases, and proposed to increase health education programs to enhance awareness of pediatric rheumatic diseases in parents. Wickwar et $\mathrm{al}^{6}{ }^{6}$ recommended a questionnaire to evaluate the knowledge level of parents about methotrexate therapy in pediatric rheumatic diseases. Although FMF is a chronic, life-long, and hereditary disease $^{4}$, there is still misinformation and a lack of knowledge about FMF. For example, $28.7 \%$ of parents were not aware that FMF is a hereditary disease, $60.8 \%$ of parents were not aware that FMF attacks do not pose a vital risk to patients, $50.3 \%$ of parents were not aware that FMF drugs are not addictive, and $53.2 \%$ of parents were not aware that FMF does not get worse as time goes on. Incorrect beliefs might occur due to the lack of education of parents. Higher education may provide easier access to information about healthier lifestyles and illnesses. In this study most of the mothers had graduated from primary school $(56.1 \%)$, while most of fathers had graduated from secondary or high school (44.4\%). We found a positive correlation between education status and the knowledge level of parents. We concluded that mothers adapt well to the idea of having a chronic disease like FMF in their children and can gain knowledge about FMF even if they have lower education status. This follows from the fact that many questions were answered correctly by the majority of the responders; for example $90.1 \%$ of parents think that colchicine is an effective drug in treating FMF, 85.4\% think that FMF is not a contagious disease, $83.6 \%$ think that FMF may progress to chronic kidney disease without regular colchicine usage, and $80.1 \%$ think that FMF must be followed by a pediatric rheumatologist and/or a pediatrician.

One of the most important attitude problems in parents is not using the therapy on time for their children with FMF. Parents' ideas about addiction and side effects of treatments and children's fears of the subcutaneous needle treatments may lead to irregular use of treatments. In the present study, half of the parents thought that FMF drugs are addictive, and $38.6 \%$ thought that FMF drugs cause infertility over time. Delayed treatment due to late diagnosis may cause the development of complications, such as amyloidosis, and enhance morbidity and mortality in patients as well as bring severe economic and psychologic burden to families and communities. ${ }^{16}$ In a society, the training of patients with chronic diseases and their caregivers could improve the 
course of these diseases and the quality of life of patients and family members. ${ }^{17}$ Patient and caregiver education aims to develop a sense of responsibility and improved health by building positive habits, such as healthy diet, regular prescription drug use, and regular followup to keep the chronic disease under control. Parents' correct perceptions and adequate knowledge about chronic diseases have an important effect on the successful management of the disease course. Programs about raising the knowledge and awareness of FMF could ensure the process of parents' acceptance of their children's disease and provide compliance with the treatments. Compliance is often a marker of patients' and parents' understanding and adaptation to a chronic disease, such as FMF. Poor compliance with colchicine use may increase attack rates of FMF. ${ }^{4}$ The $82.5 \%$ of our patients found to be compliant represent a good compliance rate for colchicine, and we did not find any significant differences in parental knowledge scores between patients with poor and good compliance with colchicine usage. Poor colchicine response and higher disease severity with attacks cause parents to be more motivated to cope with FMF. This situation causes patients' relatives to take a more effective attitude towards obtaining information about FMF. There is a significant relationship between anti-IL-1 use and the knowledge scores about FMF; therefore, using anti-IL-1 therapy as an add-on therapy in FMF might encourage parents to read and know more about FMF to take care of their children properly and to make their lives better. Kinkar et al. ${ }^{18}$ demonstrated that the parents of children who had been earlier diagnosed with epilepsy, had more knowledge about epilepsy as a chronic disease, but in our study, interestingly, there were no significant differences in evaluation of knowledge scores between ages of parents and children's age of diagnosis. Furthermore, we did not find any correlations between parental age, patients' age, patients' age at disease onset, and parental knowledge. Therefore, we concluded that the parents' efforts to learn more information about the disease did not show any difference by the age of the patients at diagnosis or by parental age.

The main limitations of this study were the small sample size from a single centre and the lack of a control group. Another limitation of this study was the questionnaire used, which is not validated and based on a previously published/validated pediatric scale.

In conclusion, having a good level of knowledge about FMF is important to increase patient compliance with treatment and, therefore, to prevent not only acute attacks but also longterm complications. However, in our study population, the knowledge about FMF among parents was unsatisfactory. The parents whose children have a severe disease course and, therefore, require anti-IL-1 add-on therapy were more knowledgeable. Educational tools, including books, on-line or live seminars, and websites giving information about FMF disease course, treatments, prognosis, and complications should be provided immediately after diagnosis and should be continuous.

\section{Ethical approval}

This study was approved by the Gazi University Medical Faculty Ethics Board (11.06.2018/456).

\section{Acknowledgments}

The authors are grateful to all participating children and their families.

\section{Author contribution}

The authors confirm contribution to the paper as follows: study conception and design: DGY, SAB, SSA, NB; data collection: DGY; analysis and interpretation of results: DGY, SAB, SSA; draft manuscript preparation: DGY, SAB, SSA, NB. All authors reviewed the results and approved the final version of the manuscript. 


\section{Source of funding}

None.

\section{Conflicts of interest}

The authors declare no conflict of interest.

\section{REFERENCES}

1. Ben-Chetrit E, Levy M. Familial Mediterranean fever. Lancet 1998; 351: 659-664. https://doi.org/10.1016/ S0140-6736(97)09408-7

2. Bernot A, Clepet C, Dasilva C, et al; French FMF Consortium. A candidate gene for familial Mediterranean fever. Nat Genet 1997; 17: 25-31. https://doi.org/10.1038/ng0997-25

3. Tunca M; Turkish FMF Study Group. Familial Mediterranean fever (FMF) in Turkey: results of a nationwide multicenter study. Medicine (Baltimore) 2005; 84: 1-11. https://doi.org/10.1097/01. md.0000152370.84628.0c

4. Onen F. Familial Mediterranean fever. Rheumatol Int 2006; 26: 489-496. https://doi.org/10.1007/s00296005-0074-3

5. Alghamdi M. Familial Mediterranean fever, review of the literature. Clin Rheumatol 2017; 36: 1707-1713. https://doi.org/10.1007/s10067-017-3715-5

6. Wickwar S, Buerkle K, McBain H, et al. Adaptation of the methotrexate in rheumatoid arthritis knowledge questionnaire (MiRAK) for use with parents of children with juvenile idiopathic arthritis: a qualitative study. Pediatr Rheumatol Online J 2013; 11: 27. https://doi.org/10.1186/1546-0096-11-27

7. Al-Eid WM, Madi SM, Bahabri SA, Al-Mayouf SM. Evaluation of parental knowledge of Pediatric Rheumatic Diseases. Saudi Med J 2001; 22: 531-536.

8. Rani A, Thomas PT. Parental knowledge, attitude, and perception about epilepsy and sociocultural barriers to treatment. J Epilepsy Res 2019; 9: 65-75. https://doi.org/10.14581/jer.19007

9. Mat MAC, Yaacob LH, Zakaria R. Parental knowledge on thalassaemia and factors associated with refusal to screen their children. Malays J Med Sci 2020; 27: 124-133. https://doi.org/10.21315/ mjms2020.27.1.13
10. Solem EP, Primiano M, McQuillen MP, Zak Goelz M. Factors associated with parental knowledge of neurofibromatosis type 1 (NF1): parental affected status and genetic counseling. J Genet Couns 2020 29: 1151-1158. https://doi.org/10.1002/jgc4.1275

11. Chan KH, Panoch J, Carroll A, Wiehe S, Cain MP, Frankel R. Knowledge gaps and information seeking by parents about hypospadias. J Pediatr Urol 2020; 16: 166.e1-166.e8. https://doi.org/10.1016/j. jpurol.2020.01.008

12. Sohar E, Gafni J, Pras M, Heller H. Familial Mediterranean fever: a survey of 470 cases and review of the literature. Am J Med 1967; 43: 227-253. https://doi.org/10.1016/0002-9343(67)90167-2

13. Ozen S, Demirkaya E, Erer B, et al. EULAR recommendations for the management of familial Mediterranean fever. Ann Rheum Dis 2016; 75: 644-651. https://doi.org/10.1136/ annrheumdis-2015-208690

14. Pras E, Livneh A, Balow JE Jr, et al. Clinical differences between North African and Iraqi Jews with familial Mediterranean fever. Am J Med Genet 1998; 75: 216-219. https://doi.org/10.1002/(SICI)10968628(19980113)75:2<216::AID-AJMG20>3.0.CO;2-R

15. Köhler BM, Lorenz HM, Blank N. IL1-blocking therapy in colchicine-resistant familial Mediterranean fever. Eur J Rheumatol 2018; 5: 230234. https://doi.org/10.5152/eurjrheum.2018.18036

16. Koşan Z, Yılmaz S, Yerli EB, Köyceğiz E. Evaluation of the burden of care and the quality of life in the parents of Turkish children with familial Mediterranean fever. J Pediatr Nurs 2019; 48: e21-e26. https://doi.org/10.1016/j.pedn.2019.05.017

17. Turan Gürhopur FD, Işler Dalgiç A. The effect of a modular education program for children with epilepsy and their parents on disease management. Epilepsy Behav 2018; 78: 210-218. https://doi. org/10.1016/j.yebeh.2017.07.048

18. Kinkar A, Alqarni D, Alghamdi A, et al. Parental knowledge, attitudes, and behaviors toward their epileptic children at King Abdulaziz University Hospital: cross-sectional study. Interact J Med Res 2020; 9: e12697. https://doi.org/10.2196/12697 